Supporting Information

\title{
Formation of Chloroform and Other Chlorinated Byproducts by Chlorination of Triclosan-Containing Antibacterial Products
}

E. Matthew Fiss, Krista L. Rule, and Peter J. Vikesland

Pages: 13

Tables S1, S2, S3

Figures S1-S8

Discussion of model simulations 
Table S1: List of various consumer products tested and their corresponding triclosan concentrations.

Product

Antibacterial Products

Dial Mountain Fresh Antibacterial Body Wash

Equate Island Spring Antibacterial Body Wash

Softsoap Antibacterial plus Real Moisturizing Lotion Hand Soap

Dial Berry Splash Antibacterial Hand Soap

Bath and Body Works Moonlight Path Antibacterial Deep Cleansing Hand Soap

Body Essence Cucumber Melon Antibacterial Hand Lotion

Ultra Palmolive Antibacterial Dish Soap

Control Products

Ultra Palmolive Oxy-Plus Dish Soap

Ultra Concentrated Natural Citrus Joy Dish Soap

Seventh Generation Natural Dish Soap

Softsoap Naturals Milk and Honey Liquid Hand Soap

Gojo Lotion Skin Cleaner
Triclosan Content

$\%$

0.15

??

0.125

??

0.3

??

0.12

0

0

0

0

0 
Table S2. Manufacturer reported composition of each soap listed in Table 1 of the manuscript. During the experimental phase of this study each soap was tested under double-blind conditions. At the conclusion of the study the ingredient lists were examined to better understand the experimental results.

\begin{tabular}{|c|c|c|c|}
\hline $\begin{array}{l}\text { II } \\
\text { triclosan } \\
\text { water } \\
\text { sodium laureth sulfate } \\
\text { cocamidopropyl betaine } \\
\text { PEG-8 } \\
\text { fragrance } \\
\text { glycerin } \\
\text { isostearam idopropyl morpholine lactate } \\
\text { polyquatemium-10 } \\
\text { cocamidopropyl PG-dimonium chloride } \\
\text { DMDM hydantoin } \\
\text { citric acid } \\
\text { tetrasodium EDTA } \\
\text { sodium chloride } \\
\text { red } 33 \\
\text { blue } 1\end{array}$ & $\begin{array}{l}\text { III } \\
\text { water } \\
\text { magnesium dodecylbenzenesulfonate } \\
\text { sodium dodecylbenzenesulfonate } \\
\text { ammonium laureth sulfate } \\
\text { lauramidopropylamine oxide } \\
\text { SD alcohol 3-A } \\
\text { sodium xylene sulfonate } \\
\text { sodium chloride } \\
\text { fragrance } \\
\text { pentasodium pentetate } \\
\text { sodium bisulfite } \\
\text { dimethylol dimethyl hydantoin }\end{array}$ & $\begin{array}{l}\text { IV } \\
\text { triclosan } \\
\text { water stearic acid } \\
\text { sunflower seed oil } \\
\text { C12-15 alkyl ethylhexanoate } \\
\text { cetyl alcohol } \\
\text { dimethicone } \\
\text { triethanolamine } \\
\text { cucumber fruit extract } \\
\text { aloe barbadensis leaf extract } \\
\text { hydrolized silk } \\
\text { panthenol (pro vitamin B5) } \\
\text { tocopheryl acetate (vitamin E) } \\
\text { retinyl palmitate (vitamin A) } \\
\text { wheat germ oil } \\
\text { DEA-cetyl phosphate } \\
\text { fragrance } \\
\text { jojoba esters } \\
\text { nylon } 12 \\
\text { glyceryl stearate } \\
\text { PEG-100 stearate } \\
\text { polyacrylamide } \\
\text { C13-14 isoparaffin } \\
\text { laureth-7 } \\
\text { carbomer } \\
\text { xanthan gum } \\
\text { BHT } \\
\text { phenoxyethanol } \\
\text { methylparaben } \\
\text { ethylparaben } \\
\text { propylparaben } \\
\text { butylparaben } \\
\text { ultramarines } \\
\text { FD\&C yellow \#5 }\end{array}$ & $\begin{array}{l}\mathbf{V} \\
\text { triclosan } \\
\text { water } \\
\text { sodium laureth sulfate } \\
\text { cocamidopropyl betaine } \\
\text { petrolatum } \\
\text { dimethicone } \\
\text { lauric acid } \\
\text { decyl glucoside } \\
\text { acrylates/C10-30 akyl acrylate crosspolymer } \\
\text { hydroxypropyl methylcellulose } \\
\text { fragrance } \\
\text { triethanolamine } \\
\text { DMDM hydantoin } \\
\text { polyquaternium-39 } \\
\text { tetrasodium EDTA } \\
\text { polyquaternium-7 } \\
\text { D\&C orange \#4 } \\
\text { FD\&C red \#40 }\end{array}$ \\
\hline $\begin{array}{l}\mathrm{VI} \\
\text { water } \\
\text { sodium C14-16 olein sulfonate } \\
\text { lauramide DEA } \\
\text { glycol stearate } \\
\text { sodium chloride } \\
\text { cocamidopropyl betaine } \\
\text { fragrance } \\
\text { DMDM hydantoin } \\
\text { polyquaternium-7 } \\
\text { citric acid } \\
\text { aloe barbadensis gel } \\
\text { tetrasodium EDTA } \\
\text { honey } \\
\text { lactose } \\
\text { milk protein } \\
\text { glycerin } \\
\text { silk peptide } \\
\text { hydrolized silk protein } \\
\text { D\&C yellow \#10 } \\
\text { FD\&C red \#40 }\end{array}$ & $\begin{array}{l}\text { VII } \\
\text { triclosan } \\
\text { water } \\
\text { sodium trideceth sulfate } \\
\text { hydrogenated soybean oil } \\
\text { sodium lauroamphoacetate } \\
\text { petrolatum } \\
\text { cocamide MEA } \\
\text { glycerin } \\
\text { ammonium chloride } \\
\text { fragrance } \\
\text { propylene glycol } \\
\text { tocopheryl acetate (vitamin E acetate) } \\
\text { artemia extract } \\
\text { echinacea purpurea (coneflower) extract } \\
\text { butyrospermum parkii (shea butter) } \\
\text { cellulose } \\
\text { hydroxypropyl methylcellulose } \\
\text { guar hydroxypropyltrim onium chloride } \\
\text { tetinyl palmitate (vitamin A palmitate) } \\
\text { benzophenone-4 } \\
\text { citric acid } \\
\text { imidazolidinyl urea } \\
\text { DMDM hydantion } \\
\text { tetrasodium EDTA } \\
\text { mica } \\
\text { titanium dioxide } \\
\text { ultramaries } \\
\text { yellow } 5 \\
\text { red } 4\end{array}$ & $\begin{array}{l}\text { VIII } \\
\text { water } \\
\text { sodium laureth sulfate } \\
\text { sodium lauryl sulfate } \\
\text { cocamide DEA } \\
\text { buttlyene glycol } \\
\text { citric acid } \\
\text { cocamidopropyl betaine } \\
\text { glycol distearate } \\
\text { lauramide DEA } \\
\text { soyamide DEA } \\
\text { soyamidopropyl betaine } \\
\text { tetrasodium EDTA } \\
\text { ammonium chloride } \\
\text { fragrance } \\
\text { DMDM hydantoin } \\
\text { iodopropynyl butylcarbamate } \\
\text { methylchloroisothiazolinone } \\
\text { methylisothiazolinone } \\
\text { red } 33\end{array}$ & $\begin{array}{l}\text { IX } \\
\text { triclosan } \\
\text { water } \\
\text { ammonium C12-15 pareth sulfate } \\
\text { magnesium dodecylbenzene sulfonate } \\
\text { lauramidopropylamine oxide } \\
\text { SD alcohol 3-A } \\
\text { sodium xylenesulfonate } \\
\text { sodium chloride } \\
\text { fragrance } \\
\text { pentasodium pentetate } \\
\text { DMDM hydantoin } \\
\text { sodium bisulfite } \\
\text { D\&C orange \#4 }\end{array}$ \\
\hline $\mathrm{X}$ & $\mathrm{XI}$ & XII & XIII \\
\hline $\begin{array}{l}\text { water } \\
\text { magnesium dodecylbenzenesulfonate } \\
\text { sodium dodecylbenzenesulfonate } \\
\text { ammonium laureth sulfate } \\
\text { lauramidopropylamine oxide } \\
\text { SD alcohol 3-A } \\
\text { sodium xylene sulfonate } \\
\text { sodium chloride } \\
\text { fragrance } \\
\text { pentasodium pentetate } \\
\text { sodium bisulfite } \\
\text { dimethylol dimethyl hydantoin }\end{array}$ & $\begin{array}{l}\text { water } \\
\text { sodium lauryl sulfate } \\
\text { sodium pareth } 23 \text { sulfate } \\
\text { SD alcohol } 40 \mathrm{~B} \\
\text { C12-14-16 dimethyl amine oxide } \\
\text { undeceth-9 } \\
\text { sodium lauryl benzene sulfonate } \\
\text { sodium chloride } \\
\text { cyclohexanediamine } \\
\text { PPG-26 } \\
\text { polyacetate } \\
\text { fragrance } \\
\text { yellow } 5 \\
\text { red } 33\end{array}$ & $\begin{array}{l}\text { triclosan } \\
\text { water } \\
\text { sodium laureth sulfate } \\
\text { decyl glucoside } \\
\text { cocamidopropyl betaine } \\
\text { sodium chloride } \\
\text { cocamide MEA } \\
\text { fragrance } \\
\text { PEG-120 methyl glucose dioleate } \\
\text { PEG-18 glyceryl oleate/cocoate } \\
\text { aloe barbadensis leaf juice } \\
\text { polyquaternium-7 } \\
\text { DMDM hydantoin } \\
\text { tetrasodium EDTA } \\
\text { citric acid } \\
\text { sodium sulfate } \\
\text { red } 33 \\
\text { red } 4\end{array}$ & $\begin{array}{l}\text { triclosan } \\
\text { water } \\
\text { sodium laureth sulfate } \\
\text { cocamidopropyl betaine } \\
\text { PET-8 } \\
\text { glycerin } \\
\text { disodium laureth sulfosuccinate } \\
\text { fragrance } \\
\text { polyquaternium-10 } \\
\text { PEG-7 glyceryl cocoate } \\
\text { DMDM hydantoin } \\
\text { idopropynyl butyl carbam ate } \\
\text { citric acid } \\
\text { tetrasodium EDTA } \\
\text { FD\&C blue \#1 } \\
\text { D\&C red \#33 }\end{array}$ \\
\hline XIV & $\mathrm{XV}$ & $\mathrm{XVI}$ & XVII \\
\hline $\begin{array}{l}\text { triclosan } \\
\text { water } \\
\text { ammonium C12-15 pareth sulfate } \\
\text { magnesium dodecylbenzene sulfonate } \\
\text { lauramidopropylamine oxide } \\
\text { SD alcohol 3-A } \\
\text { sodium xylenesulfonate } \\
\text { sodium chloride } \\
\text { fragrance } \\
\text { pentasodium pentetate } \\
\text { DMDM hydantoin } \\
\text { sodium bisulfite } \\
\text { D\&C orange \#4 }\end{array}$ & $\begin{array}{l}\text { Water } \\
\text { natural fragrance (citrus oil) } \\
\text { preservative } \\
\text { corn and/or coconut-based surfactants }\end{array}$ & $\begin{array}{l}\text { triclosan } \\
\text { water } \\
\text { sodium laureth sulfate } \\
\text { cocamidopropyl betaine } \\
\text { petrolatum } \\
\text { dimethicone } \\
\text { lauric acid } \\
\text { decyl glucoside } \\
\text { acylates/C10-30 akyl acrylate crosspolymer } \\
\text { hydroxypropyl methylcellulose } \\
\text { fragrance } \\
\text { triethanolamine } \\
\text { DMDM hydantoin } \\
\text { polyquaternium-39 } \\
\text { tetrasodium EDTA } \\
\text { polyquaternium-7 } \\
\text { D\&C orange \#4 } \\
\text { FD\&C red \#40 }\end{array}$ & $\begin{array}{l}\text { triclosan } \\
\text { water } \\
\text { sodium laureth sulfate } \\
\text { cocamidopropyl betaine } \\
\text { PEG-8 } \\
\text { fragrance } \\
\text { glycerin } \\
\text { isostearam idopropyl morpholine lactate } \\
\text { polyquatemium-10 } \\
\text { cocamidopropyl PG-dimonium chloride } \\
\text { DMDM hydantoin } \\
\text { citric acid } \\
\text { tetrasodium EDTA } \\
\text { sodium chloride } \\
\text { red } 33 \\
\text { blue } 1\end{array}$ \\
\hline
\end{tabular}




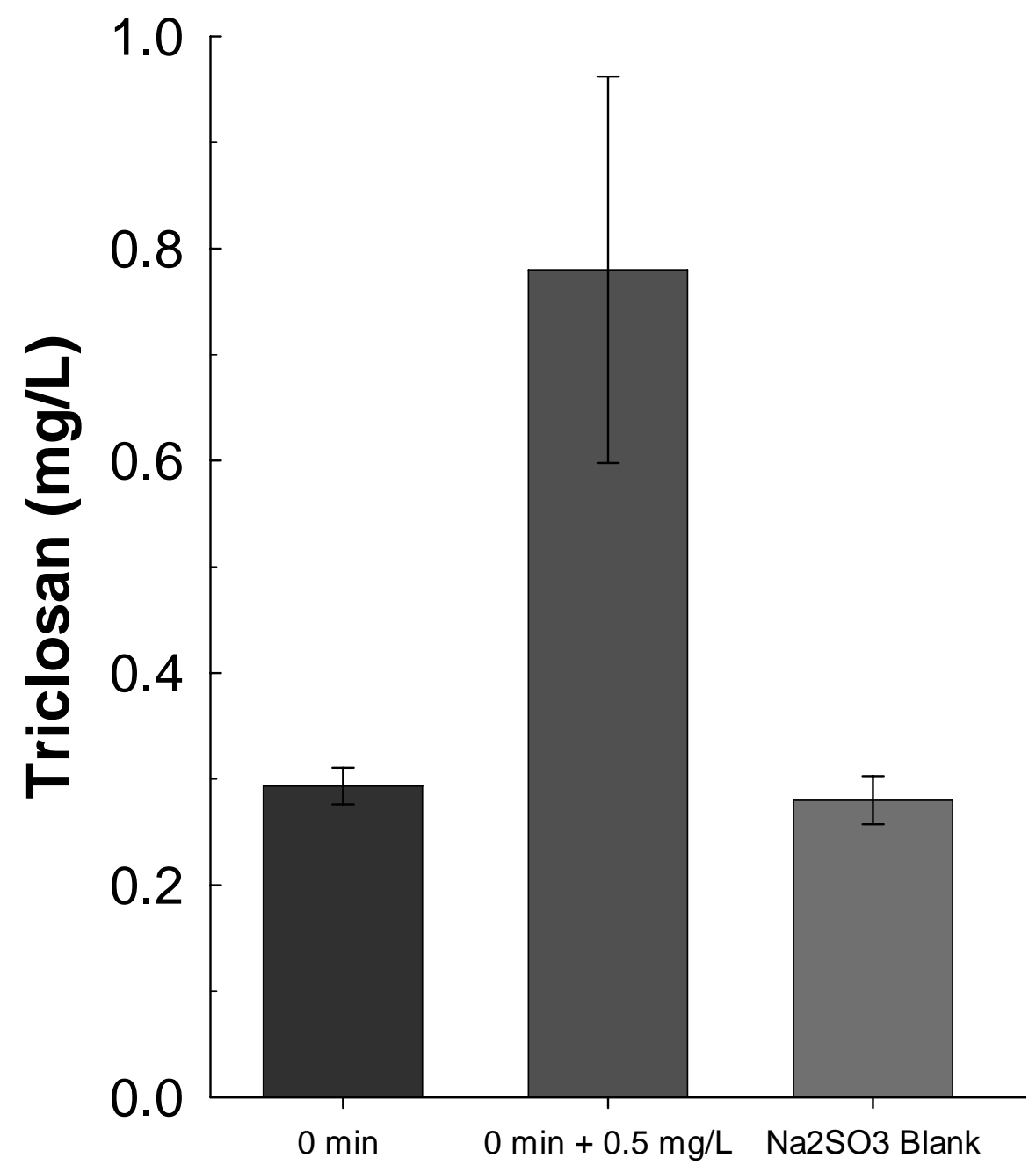

Figure S1: In order to establish the validity of the PFBBr technique for triclosan quantification, a control spike recovery experiment was performed where $0.5 \mathrm{mg} / \mathrm{L}$ of triclosan was added to a soap solution initially containing $0.30 \mathrm{mg} / \mathrm{L}$ of triclosan and the amount of triclosan recovered was recorded. A second comparison was made to a soap sample when an equivalent aliquot of sodium sulfite solution was added. This experiment showed that the spiked triclosan was completely recovered and that sodium sulfite had no affect on the recovery amount. Error bars reflect the standard deviation of triplicate samples. 


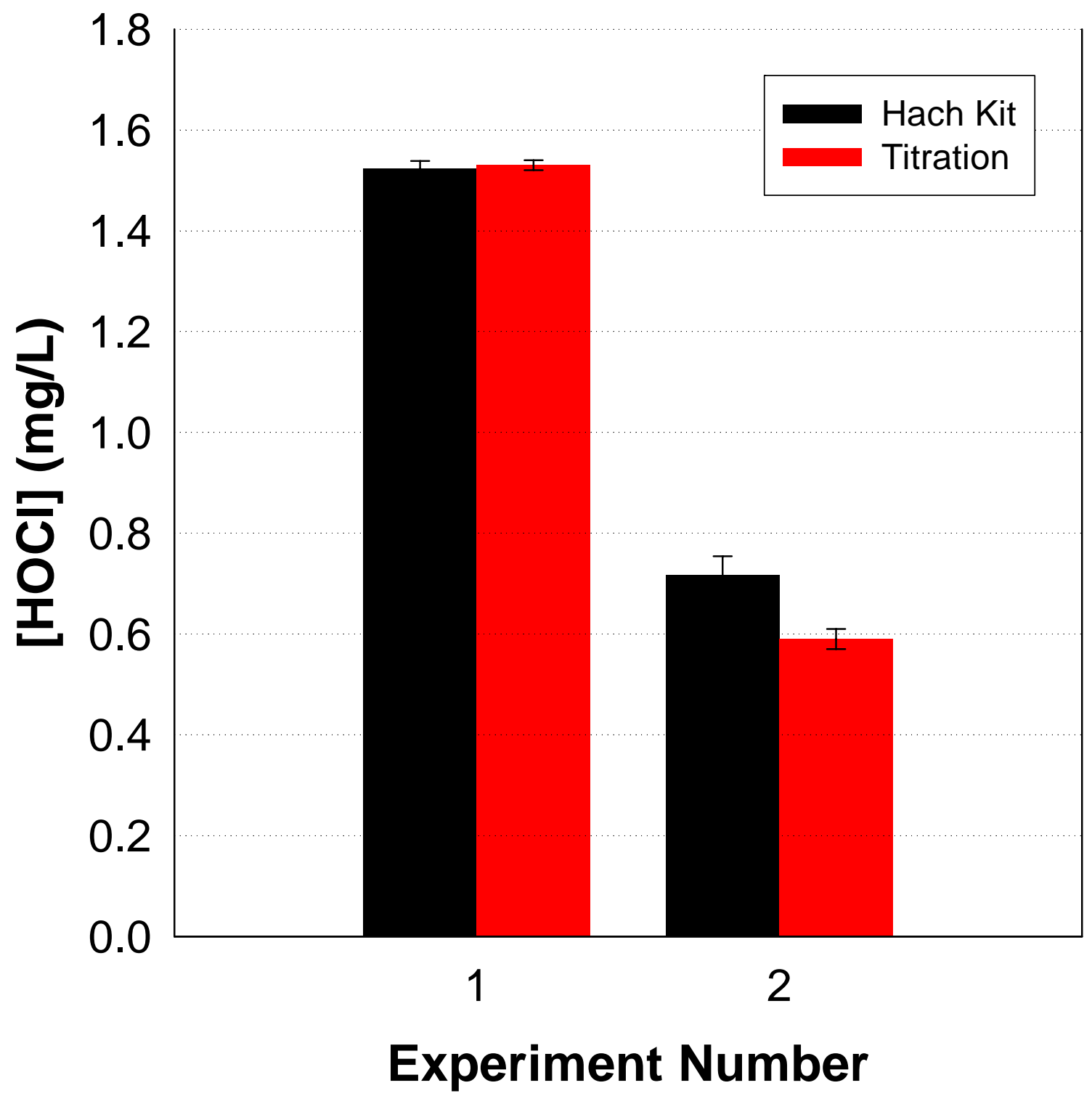

Figure S2: Comparison between field chlorine Hach kit and manual DPD-FAS titrations performed in the lab. 


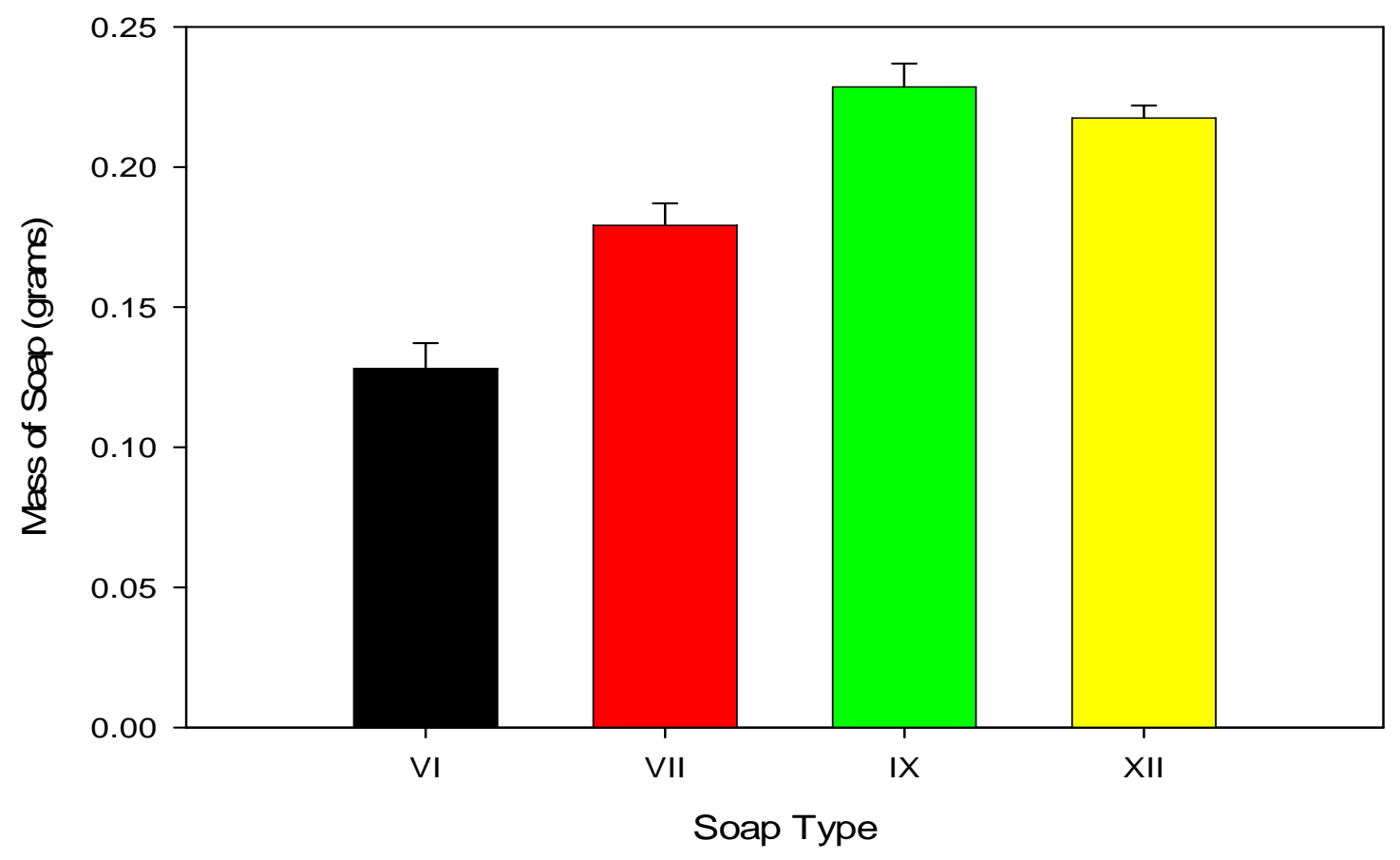

Figure S3: Mass of each tested soap dispensed during field experiments by transferring a given aliquot of soap to the reaction vial. Each mass was distributed using an auto-pipettor and can be compared to the $0.25 \mathrm{~g}$ mass of soap utilized in the laboratory experiments. Product formation levels were normalized to a level of $0.25 \mathrm{~g} / \mathrm{L}$ in order to allow for comparisons between other experiments. 


\section{Discussion of model proposed by Rule et al to predict consumption rates of free chlorine and triclosan during reactions at $25^{\circ} \mathrm{C}$.}

Rule et al (1) developed the model shown below to predict the observed rates of the $\mathrm{pH}$ dependent reaction between triclosan and free chlorine. The reaction rates are highest between a $\mathrm{pH}$ of 7 to 9, which also happens to be the $\mathrm{pH}$ range that encompasses the majority of drinking waters.

$$
\frac{d[\mathrm{HOCl}]_{\text {Total }}}{d t}=\frac{d[\text { triclosan }]_{\text {Total }}}{d t}=-k_{A r O^{-}}[\text {phenolate }- \text { triclosan }][\mathrm{HOCl}]
$$

where

$$
\text { triclosan } \leftrightarrow \text { phenolate }- \text { triclosan }+H^{+} \quad K_{A}=10^{-7.9}
$$

and

$$
\mathrm{HOCl} \leftrightarrow \mathrm{OCl}^{-}+\mathrm{H}^{+} \quad \mathrm{K}_{\mathrm{HOCl}}=10^{-7.5}
$$

The model can then be expressed as the pseudo first order equation

$$
\frac{d[\text { triclosan }]_{\text {total }}}{d t}=k_{\text {obs }}[\text { triclosan }]_{\text {total }}
$$

where

$$
k_{o b s}=-k_{A r O^{-}}\left(\frac{1}{1+\frac{\left[H^{+}\right]}{K_{A}}}\right)[H O C l]_{\text {initial }} \quad \quad \mathrm{k}_{\mathrm{ArO}^{-}}=5.40 \times 10^{3} \mathrm{M}^{-1} \mathrm{~S}^{-1}
$$

when excess free chlorine is present. Using this simplification of the model, it was possible to predict triclosan concentration as a function of time (Figure S4). 


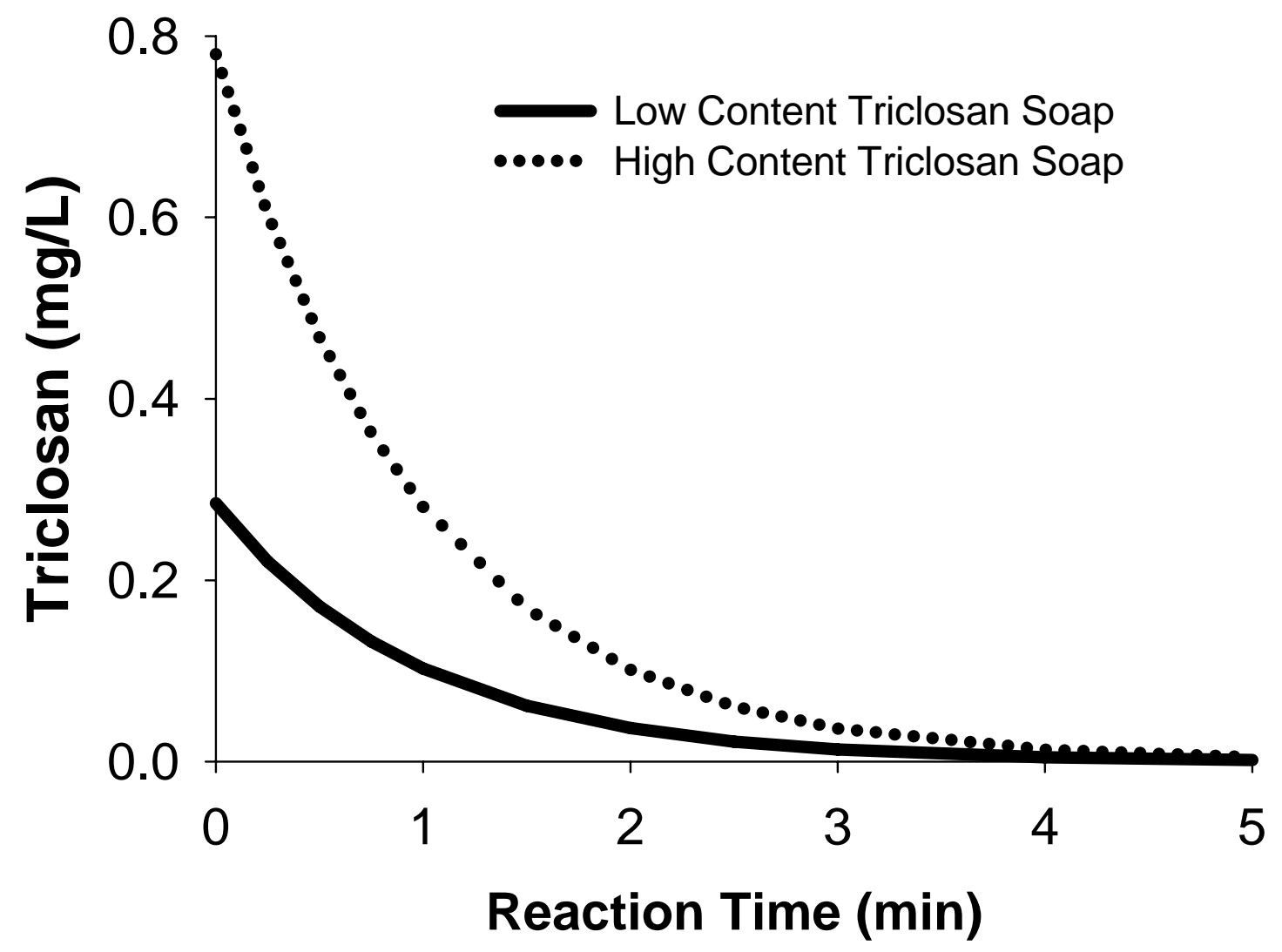

Figure S4: Triclosan consumption over time as predicted using the Rule et al. model. The high and low content soap solutions have initial triclosan concentrations of 0.78 and $0.285 \mathrm{mg} / \mathrm{L}$, respectively. 


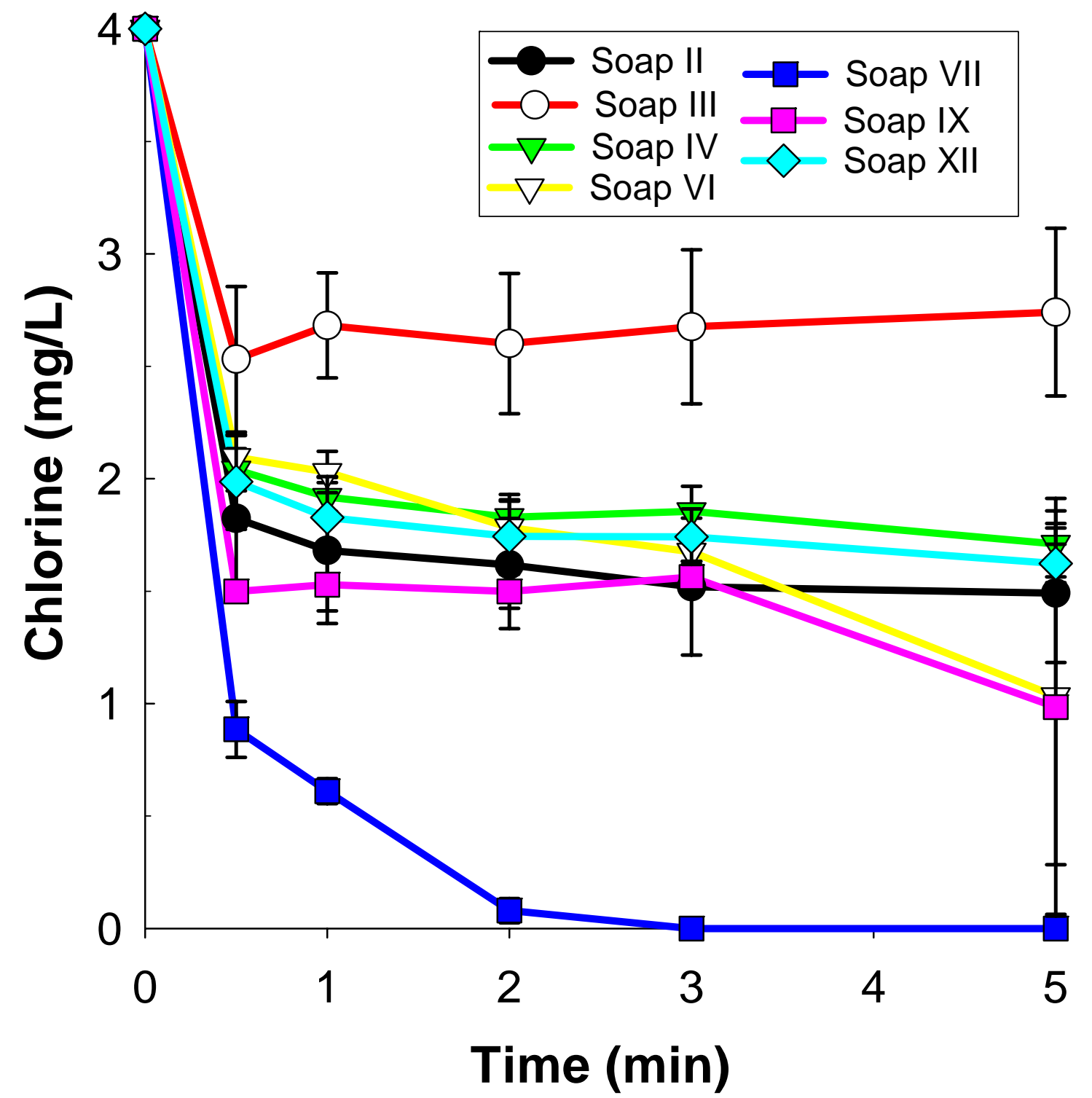

Figure S5: Chlorine consumption when several unknown product solutions are contacted with free chlorine (triclosan-containing products represented by solid symbols; triclosan-free products designated by hollow symbols). Conditions: $[\text { Free chlorine }]_{\text {initial }}=4.0 \mathrm{mg} / \mathrm{L}$ as $\mathrm{Cl}_{2},\left[\mathrm{NaHCO}_{3}\right]=2 \mathrm{mM}$, [Soap] $=0.25 \mathrm{~g} / \mathrm{L}, \mathrm{pH}=$ 7.0, $\mathrm{T}=40^{\circ} \mathrm{C}$. 


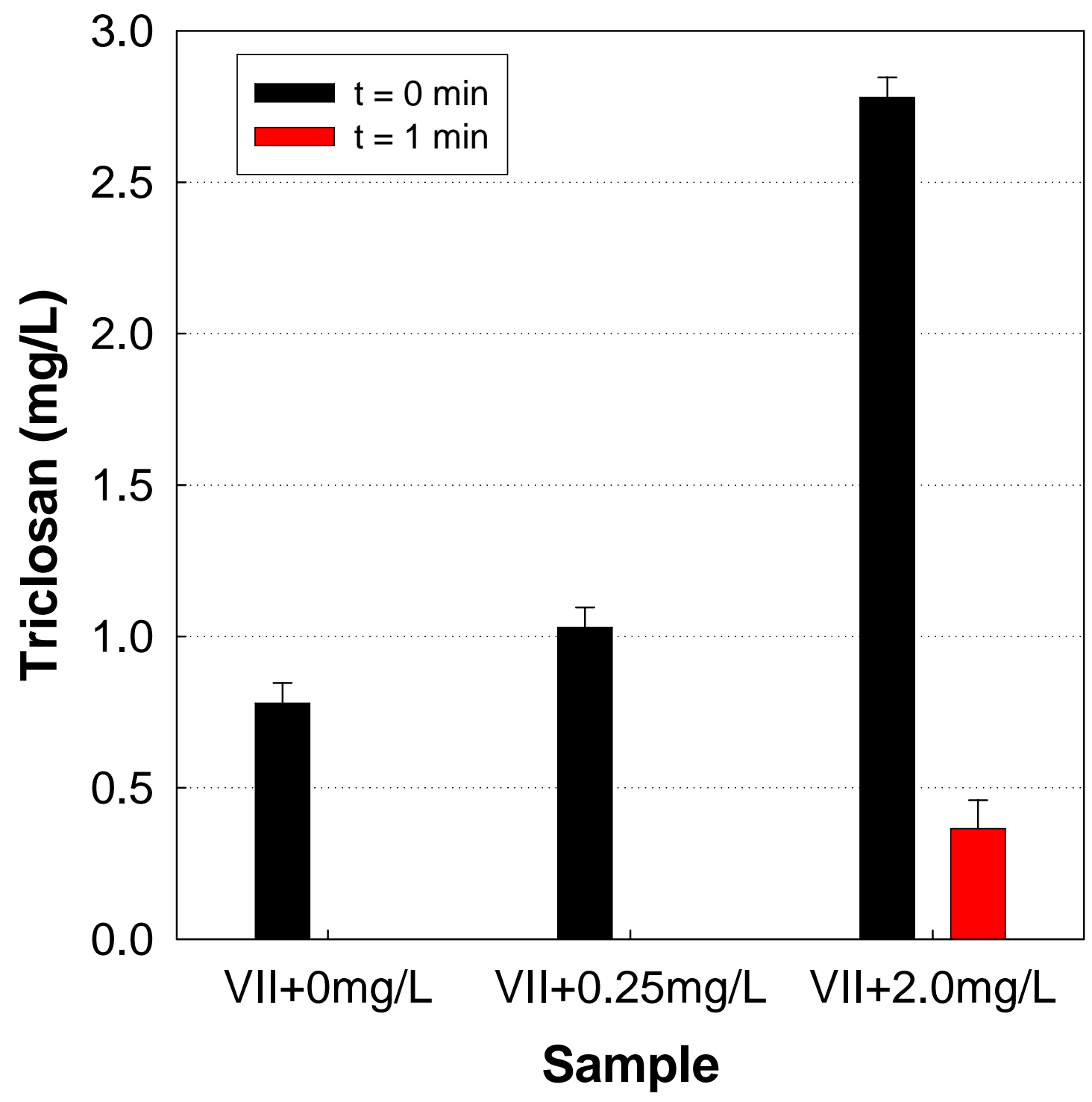

Figure S6: Initial triclosan concentration compared with triclosan concentrations after 1 minute of reaction time for soap VII solutions spiked with varying additional triclosan concentrations. Conditions: [Free chlorine $]_{\text {initial }}=2.0 \mathrm{mg} / \mathrm{L}$, [Soap] $=0.25 \mathrm{~g} / \mathrm{L}, \mathrm{pH}=7.0, \mathrm{~T}=40^{\circ} \mathrm{C}$. Triclosan only remained after 1 minute when $2.0 \mathrm{mg} / \mathrm{L}$ of triclosan was added and free chlorine was completely consumed in all cases for the reaction period. 


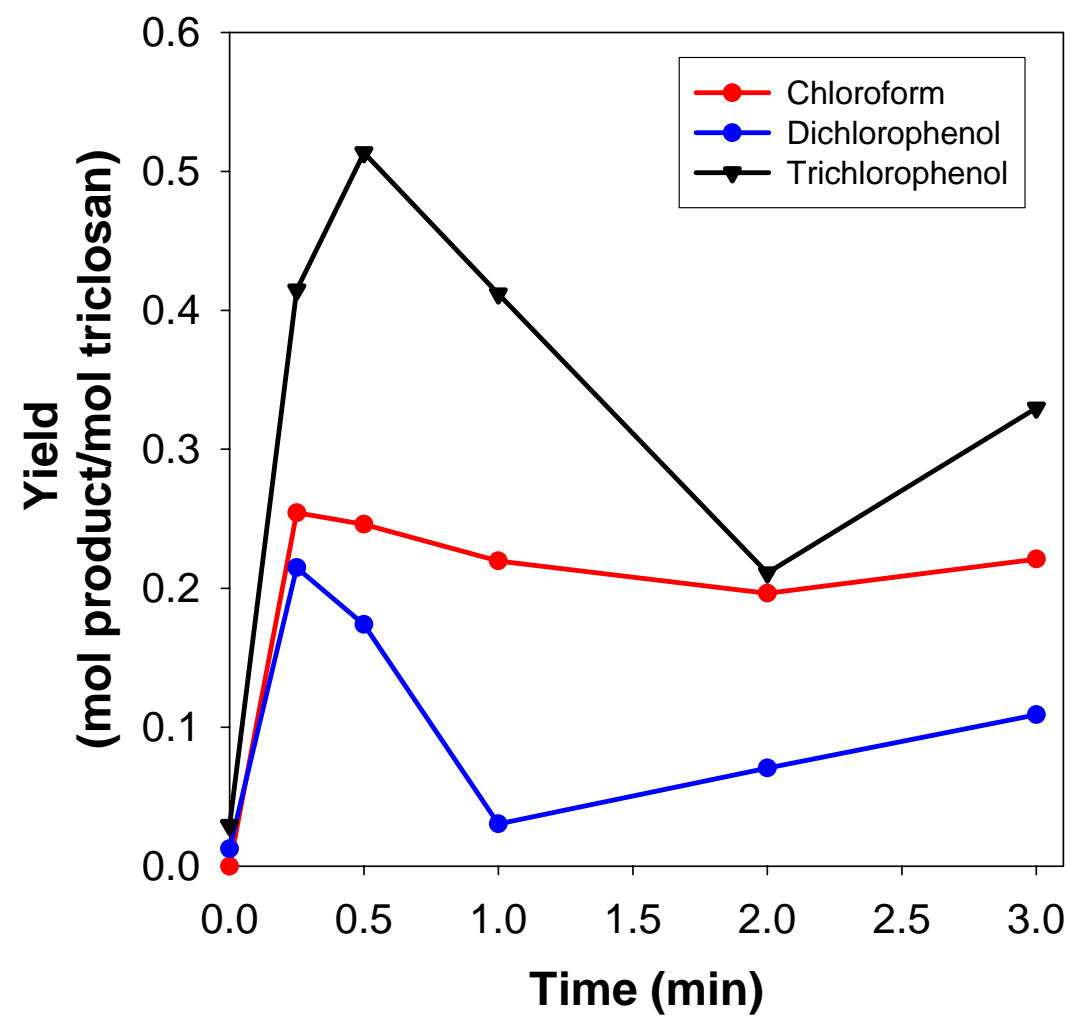

(i)

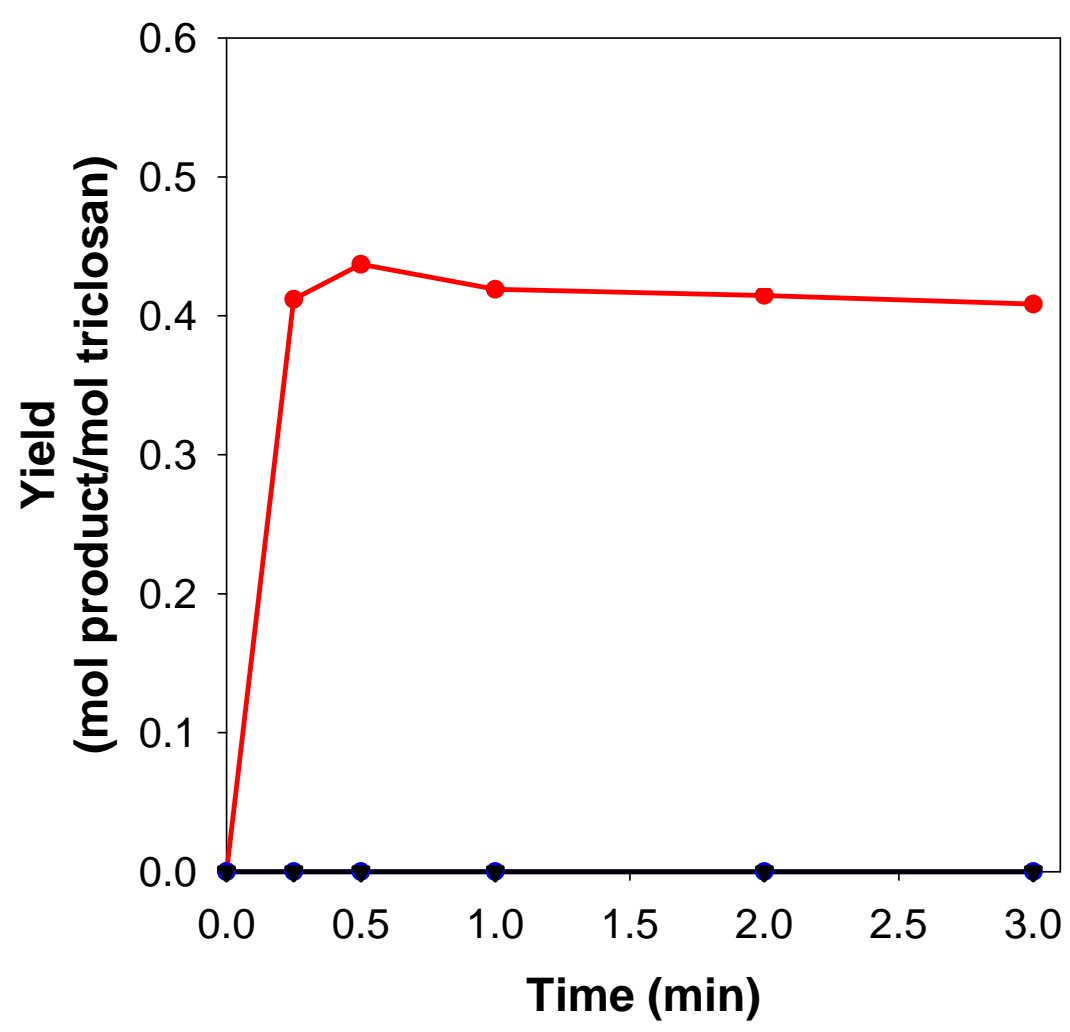

(ii)

Figure S7: Chloroform, 2,4-dichlorophenol, and 2,4,6-trichlorophenol formation yields in pure triclosan experiments with (i) $[\text { triclosan }]_{\mathrm{i}}=0.780 \mathrm{mg} / \mathrm{L}\left(=10.47 \mu \mathrm{M}\right.$ free chlorine $\mu \mathrm{M}$ triclosan) and (ii) $[\text { triclosan }]_{\mathrm{i}}=0.285 \mathrm{mg} / \mathrm{L}(=28.66 \mu \mathrm{M}$ free chlorine $/ \mu \mathrm{M}$ triclosan). Experiments conducted with [triclosan] $]_{i}=0.285 \mathrm{mg} / \mathrm{L}$ did not have any chlorophenols detected during the reaction time. Conditions: [Free chlorine $]_{i}=2.0 \mathrm{mg} / \mathrm{L}$ as $\mathrm{Cl}_{2},\left[\mathrm{NaHCO}_{3}\right]=2 \mathrm{mM}, \mathrm{pH}=7.0$. 


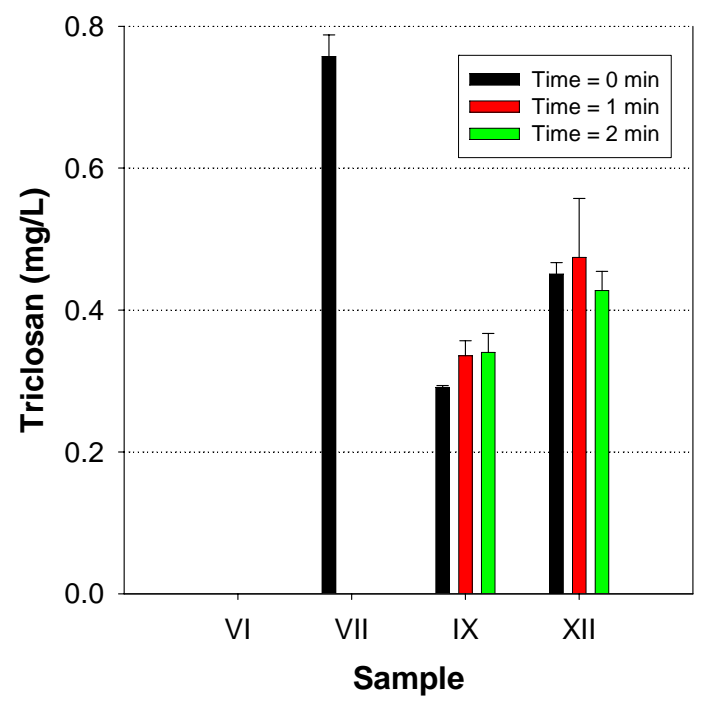

(i)

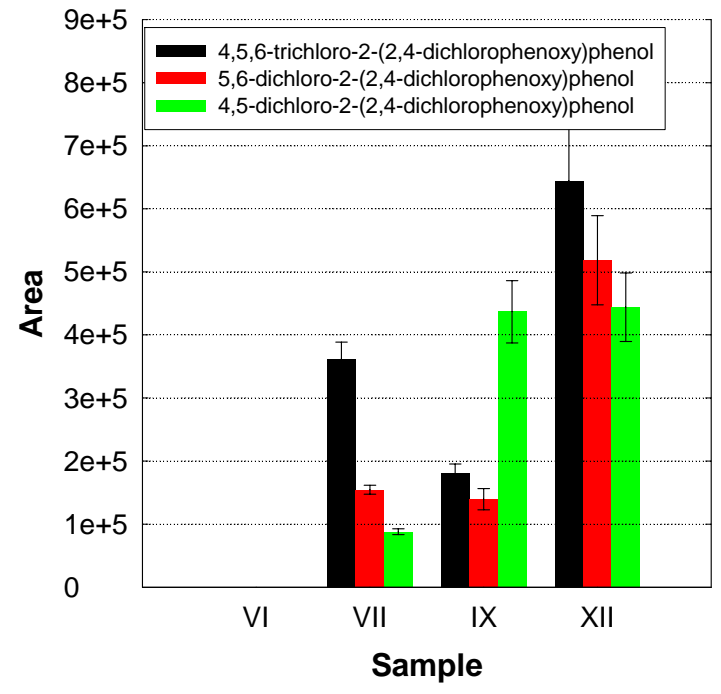

(iii)

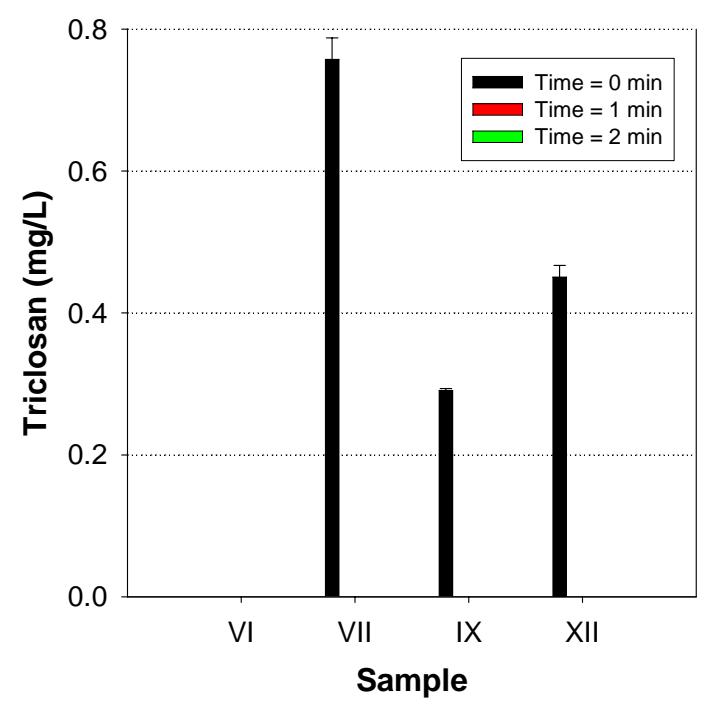

(ii)

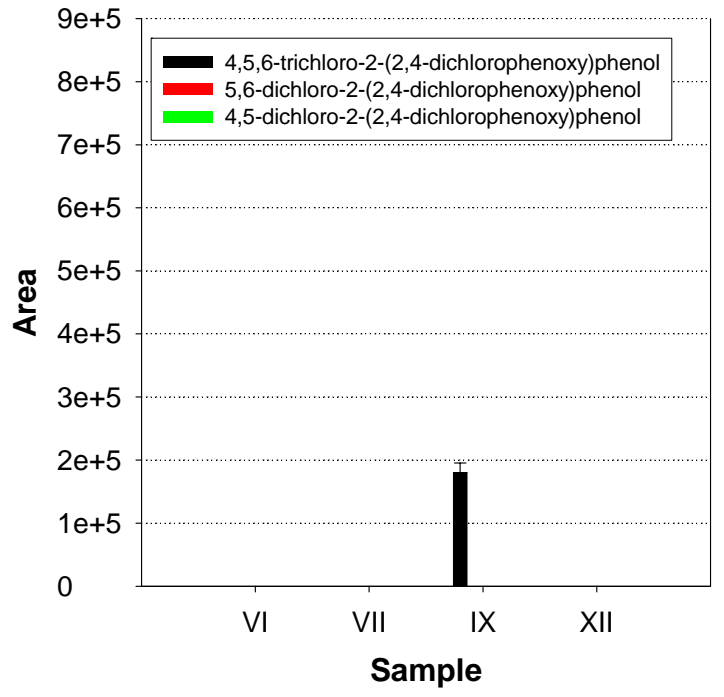

(iv)

Figure S8: Triclosan consumption and (chlorophenoxy)phenol formation in Atlanta and Danville experiments: (i) = triclosan consumption in Atlanta, (ii) = triclosan consumption in Danville, (iii) intermediate formation in Atlanta, (iv) = intermediate formation in Danville. 
Table S3: Breakdown of ICP analysis for Atlanta and Danville tap waters.

\begin{tabular}{|c|c|c|}
\hline Constituent & Concentration in Atlanta water (ppb) & Concentration in Danville water (ppb) \\
\hline $\mathrm{Mg}$ & 1305.0 & 2196.0 \\
\hline Al" & 9.8 & 178.6 \\
\hline S̄i & 3314.0 & 6786.0 \\
\hline $\mathrm{P}$ & 109.8 & 263.1 \\
\hline $\mathrm{K}$ & 16990.0 & 2547.0 \\
\hline $\mathrm{Ca}$ & 4834.0 & 11540.0 \\
\hline $\mathrm{Cr}$ & 0.3 & 0.6 \\
\hline $\mathrm{Fe}$ & 16.1 & 79.4 \\
\hline $\mathrm{Mn}$ & 0.9 & 2.0 \\
\hline $\mathrm{Ni}$ & 1.0 & 2.0 \\
\hline $\mathrm{Cu}$ & 117.6 & 12.6 \\
\hline $\mathrm{Zn}$ & 59.0 & 37.5 \\
\hline As & 0.1 & 1.2 \\
\hline Sn & 0.1 & 6.9 \\
\hline Cd & 0.2 & 1.9 \\
\hline $\mathrm{Pb}$ & 0.3 & 8.0 \\
\hline
\end{tabular}

References cited.

(1) Rule, K. L.; Ebbett, V. R.; Vikesland, P. J. Formation of chloroform and chlorinated organics by free-chlorine-mediated oxidation of triclosan. Environ. Sci. Technol. 2005, 39, 3176-3185. 